\title{
High reflectivity intracavity Bragg mirrors for the far-infrared p-Ge laser
}

\author{
Todd W. Du Bosq, Andrei V. Muravjov, Robert E. Peale \\ Department of Physics, University of Central Florida, Orlando FL, 32816
}

\begin{abstract}
Multi-layer mirrors capable of $>99.9 \%$ reflectivity at $\sim 100 \mu \mathrm{m}$ wavelengths were constructed using thin silicon etalons separated by empty gaps. Due to the large difference between the index of refraction of silicon (3.384) and vacuum (1), calculations indicate that only three periods are required to produce $99.9 \%$ reflectivity. The mirror was assembled from high purity silicon wafers, with resistivity over $4000 \mathrm{ohm}-\mathrm{cm}$ to reduce free carrier absorption. Wafers were double side polished with faces parallel within 10 arc seconds. The multi-layer mirror was demonstrated as a cavity mirror for the far-infrared p-Ge laser.
\end{abstract}

Keywords: Far-infrared, Terahertz, p-Germanium, Bragg mirror

\section{INTRODUCTION}

Study and monitoring of atmospheric constituents is important for both security and environmental reasons. To detect certain molecular species at low concentrations requires new technologies to improve accuracy. Optical techniques are particularly attractive because they are based on rich and characteristic electromagnetic molecular spectra that span UV to microwave frequencies. The far-infrared region of the electromagnetic spectrum is rich in fingerprint bands that have been underutilized for lack of adequate instrumentation. Recently developed coherent sources operating from 10 to beyond $100 \mu \mathrm{m}$ enable new methods of ultra-sensitive molecular detection.

Intensity-based absorption measurements using lasers provide poor sensitivity for molecular sensing due to unavoidable and substantial intensity instabilities. Cavity ring-down spectroscopy (CRDS) is a fairly new method that provides immunity from laser pulse intensity variations and gives a significant signal-to-noise advantage over intensity -based methods. ${ }^{1}$ Absorption coefficients are determined by monitoring the change in the decay time of the circulating photon pulse when the absorbing species is present. The extraordinary sensitivity, which can be better than parts per billion, of a cavity ring-down spectrometer is realized by achieving multi-kilometer path lengths for a laser pulse inside a high Q-factor cavity. However, no cavity ring-down spectrometer ${ }^{2}$ has been demonstrated at wavelengths longer than $8.5 \mu \mathrm{m}$.

The inexistence of a far-infrared CRDS can be attributed to lack of a sufficiently high-Q cavity, which is required for achieving an adequately long cavity ring down time. High reflectivity mirrors are required to provide adequately long decay time $\tau_{0}=\mathrm{t}_{\mathrm{r}} / 2(1-\mathrm{R})$, where $\mathrm{t}_{\mathrm{r}}$ is the cavity round-trip time. A mirror reflectivity $\mathrm{R}$ of $99.9 \%$ gives a $1.7 \mu$ s decay time, sufficiently long for experimental convenience, for a cavity length $\mathrm{L}=0.5 \mathrm{~m}$. In the visible and near IR, such high $\mathrm{R}$ values are readily provided by multi-layer dielectric mirrors, typically formed from evaporated glassy layers of materials such as $\mathrm{SiO}_{2}, \mathrm{TiO}_{2}, \mathrm{ZrO}_{2}$, and $\mathrm{ThF}_{4}$. However, such materials, and most other glasses and polymers, will not work in the far-IR due to high absorption loss. Moreover, homogeneous metal films that are thick enough to provide high reflectivity have high loss due to free carrier absorption, which increases as the square of the wavelength.

Far-IR Fabry-Perot (FP) cavities have been constructed using metal mesh mirrors. ${ }^{3}$ Reports of Q values of $\sim 100$ are typical for such cavities. Since 


$$
Q=q F=\frac{q \pi \sqrt{R}}{1-R}
$$

where $\mathrm{q}$ is the resonance order, this implies an achieved reflectivity for mesh mirrors of only about $95 \%$. This is worse than the $99 \%$ reflectivity expected for gold films, even though theoretically mesh mirrors should have lower loss. In other words, no experiment has thus far demonstrated far-IR mirrors with reflectivity sufficiently high for CRDS.

This paper describes innovative high-reflectivity mirrors that might be further developed for far-IR CRDS applications. The innovation is to form alternating layers of thin silicon etalons separated by vacuum gaps. ${ }^{4}$ Silicon has high far-IR transparency in the region 10-1000 $\mu \mathrm{m}$, except in relatively narrow bands where phonon absorption occurs. At room temperature, phonon absorption amounts to approximately $0.01 \mathrm{~cm}^{-1}$ at wavelengths longer than 50 $\mu \mathrm{m}$. Free carrier absorption losses for intrinsic Si at room temperature are also about $0.01 \mathrm{~cm}^{-1}$. Because of the large difference in index of refraction between silicon (3.384) and air (1), only three periods are required to produce $99.9 \%$ reflectivity. The principle was independently suggested and tested recently, ${ }^{5}$ although accurate measurements of the achieved reflectivity were lacking. This paper presents results of a test using the novel mirror as one of the cavity mirrors for a far-infrared p-Ge laser (1 W peak power, tuning range 70-200 $\mu \mathrm{m}, \mathrm{MHz}$ linewidth) ${ }^{6}$ The low gain of this laser medium $\left(0.01-0.1 \mathrm{~cm}^{-1}\right)$ puts high demands on mirror reflectivity. By comparing observed laser thresholds with results using traditional mirrors, a qualitative indication of achieved mirror reflectivity is obtained.

\section{THEORETICAL CONSIDERATIONS}

High reflectivity far-infrared multi-layer Bragg mirrors are constructed by stacking alternating thin films of different refractive index and appropriate thickness.? The reflectivity, $\mathrm{R}$, of the Bragg mirror is determined from the matrix formulation of the boundary conditions at the film interfaces found from Maxwell's equations. The amplitude reflection coefficient, $r$, of the Bragg mirror is given as,

$$
r=\frac{\eta_{m} \vec{E}_{m}-\vec{H}_{m}}{\eta_{m} \vec{E}_{m}+\vec{H}_{m}}
$$

where

$$
\left(\frac{\vec{E}_{m}}{\vec{H}_{m}}\right)=\vec{M}\left(\frac{1}{\eta_{s}}\right)
$$

$\vec{E}_{m}$ is the electric vector, $\vec{H}_{m}$ is the magnetic vector, $\eta_{m}$ is the effective refractive index of the incident medium, $\eta_{s}$ is the refractive index of the medium behind the mirror, and $\vec{M}$ is the product matrix of L layers given by

$$
\vec{M}=\vec{M}_{L} \vec{M}_{L-1} \cdots \vec{M}_{j} \cdots \vec{M}_{2} \vec{M}_{1}
$$

where $\vec{M}_{j}$ represents the $j$ th layer of the Bragg mirror given by

$$
\vec{M}_{j}=\left(\begin{array}{cc}
m_{11} & i m_{12} \\
i m_{21} & m_{22}
\end{array}\right)=\left(\begin{array}{cc}
\cos \delta_{j} & \frac{i}{\eta_{j}} \sin \delta_{j} \\
i \eta_{j} \sin \delta_{j} & \cos \delta_{j}
\end{array}\right)
$$


where

$$
\delta_{j}=\frac{2 \pi}{\lambda}\left(n_{j} d_{j} \cos \theta_{j}\right)
$$

and where $\lambda$ is the wavelength of the light and $n_{j} d_{j} \cos \theta_{j}$ is the optical thickness of the $j$ th layer for the angle of refraction $\theta_{j}$. If the light waves are at normal incidence, then $\theta_{j}=0$. In this case, $\eta_{j}=n_{j}-i k$ where $n_{j}$ is the index of refraction of the medium and $k$ is the extinction coefficient of the medium. The reflectivity is then given as

$$
R=|r|^{2}
$$

A preliminary multi-layer mirror was designed as a cavity mirror for the $\mathrm{p}$-Ge laser. This mirror is composed of just 3 periods of silicon and vacuum gaps (Figure 1). The thickness of each layer is a multiple of the quarter wavelength of the light $(\lambda / 4)$ in the material. The Si layer thickness is $24.5 \mu \mathrm{m}$ and the gap thickness is $27.6 \mu \mathrm{m}$. The refractive index of silicon at $4 \mathrm{~K}$ and $100 \mu \mathrm{m}$ wavelength ${ }^{8}$ is 3.384 . For calculations, the extinction coefficient of $\mathrm{Si}$ was taken to be $2.5 \times 10^{-5}$ based on an upper bound estimate of $0.01 \mathrm{~cm}^{-1}$ for the absorption coefficient (see below). The refractive index of vacuum is unity. The incident medium in front of the Bragg mirror is Ge with an index of refraction of 3.925 at $4 \mathrm{~K}$ and $100 \mu \mathrm{m}$ wavelength. ${ }^{8}$ The medium behind the mirror is vacuum. Small $27.6 \mu \mathrm{m}$ pieces of Si used as spacers determine the air layer thickness and ensure parallelism.

The reflectivity (Eq. 7) of the Figure 1 mirror is plotted vs. wavelength in Figure 2. The maximum reflectivity for the three period Bragg mirror is $99.9 \%$. Adding a fourth period increases the reflectivity to $99.99 \%$.

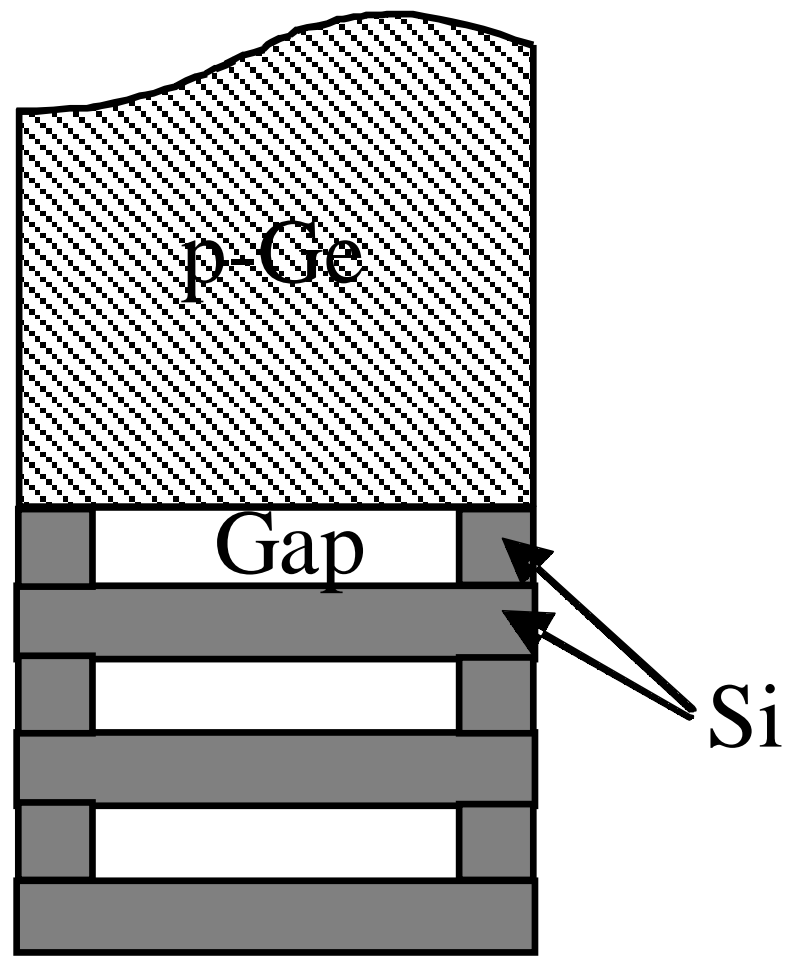

Fig. 1. Schematic drawing of the 3 period Bragg mirror based on silicon/vacuum layers for the p-Ge laser. 


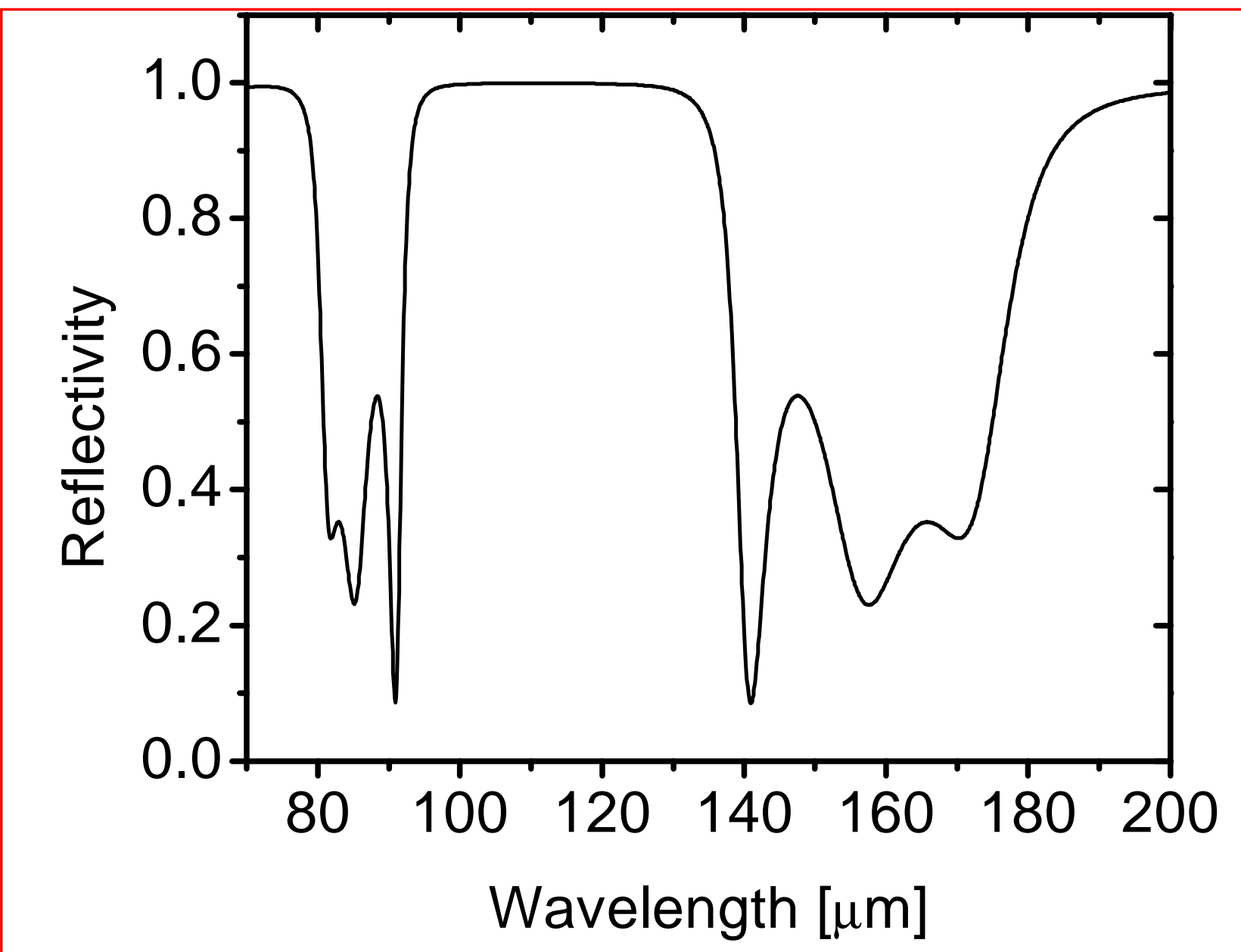

Fig. 2. Reflectivity of a three-period Bragg mirror in the emission wavelength range of the p-Ge laser. A maximum reflectivity of $99.9 \%$ is achieved.

The far-IR transmission loss of silicon single crystals is very low. Intracavity laser absorption measurements on up to $3 \mathrm{~cm}$ of silicon have been performed. ${ }^{9}$ When placed inside the cavity of a p-Ge laser at $4 \mathrm{~K}$ operating temperature, these materials have negligible effect on the laser thresholds. Since gain of the p-Ge laser is about 0.01 $\mathrm{cm}^{-1}$, while active crystal lengths are usually several $\mathrm{cm}$, the absorption coefficient of the passive semiconductor crystals at cryogenic temperatures is therefore much less than $0.01 \mathrm{~cm}^{-1}$.

\section{EXPERIMENTAL DETAILS}

High purity silicon etalons from Valley Design, with resistivity over $4000 \mathrm{ohm}-\mathrm{cm}$ to reduce free carrier absorption, are double side polished and parallel within $10 \mathrm{arc}$ seconds. The sufficiently parallel surfaces prevent beam walk-off from the cavity. The specified silicon thickness is $24.5+/-0.5 \mu \mathrm{m}$ and the gap thickness is $27.6+/-0.5$ $\mu \mathrm{m}$. The parallel gaps are produced using small $27.6 \mu \mathrm{m}$ pieces of silicon as spacers.

The multi-layer mirrors are tested as cavity back mirrors for the p-Ge laser. The silicon pieces are stacked layer by layer onto the p-Ge crystal end face. After the six alternating layers are constructed, a brass washer is placed on top of the mirror with a rubber string attaching the whole structure in place on the crystal, as shown in Figure 3. A $\mathrm{SrTiO}_{3}$ mirror smaller than the crystal end face to be used as the output mirror is attached to the other end face of the crystal with a rubber string, as shown in Figure 3. The active Ga-doped Ge crystal has dimensions $4.75 \mathrm{~mm} \times 6.75$ $\mathrm{mm} \times 35.20 \mathrm{~mm}$. The crystal ends were polished flat and parallel within 30 arc seconds. The electric field was generated in the crystal by applying voltage using a thyratron pulser to ohmic contacts (Figure 3). The magnetic field 
was supplied by a superconducting solenoid. A liquid-helium cooled Ge:Ga photoconductor inside the cryostat detects the radiation. The laser operation zones, the electric and magnetic fields in which the laser operates, are recorded for two different end mirrors. Laser thresholds of the Bragg end mirror are compared with those of a $\mathrm{SrTiO}_{3}$ end mirror to obtain a qualitative indication of mirror reflectivity.
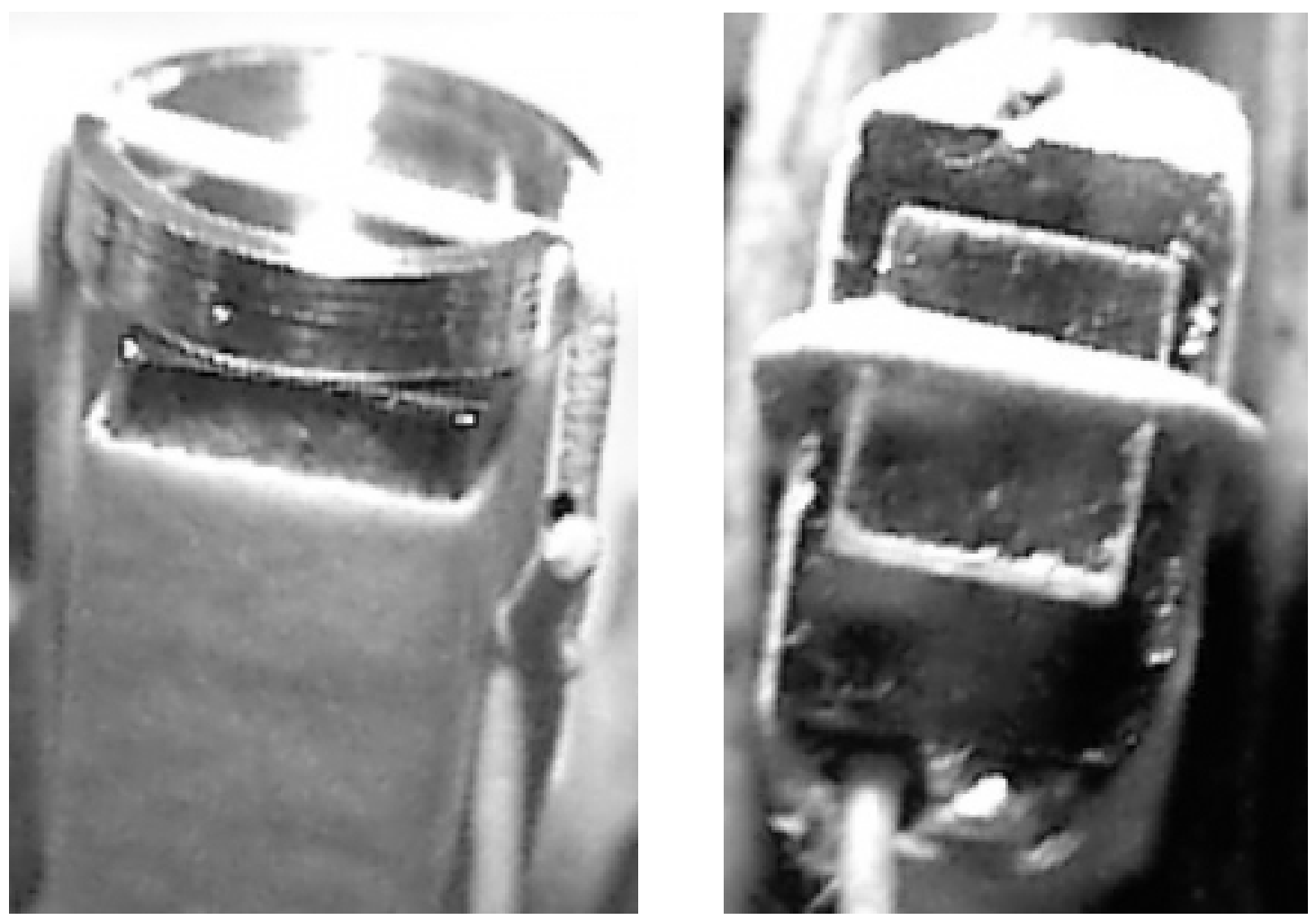

Fig. 3. (left) Photograph of the Bragg mirror on the end of a p-Ge laser crystal held in place with a brass washer and a rubber string. (Right) Photograph of the $\mathrm{SrTiO}_{3}$ output mirror on the end of a p-Ge laser held on with a rubber string. Wires providing the electric field to the ohmic contacts are on the lateral sides of the crystal.

\section{EXPERIMENTAL RESULTS}

Figure 4 shows the laser operation zones in the space of the applied $\mathbf{E}$ and $\mathbf{B}$ fields. The zone using the Bragg mirror is compared to the same laser with a $\mathrm{SrTiO}_{3}$ back mirror. The electric field threshold using $\mathrm{SrTiO}_{3}$ mirror is lower. 


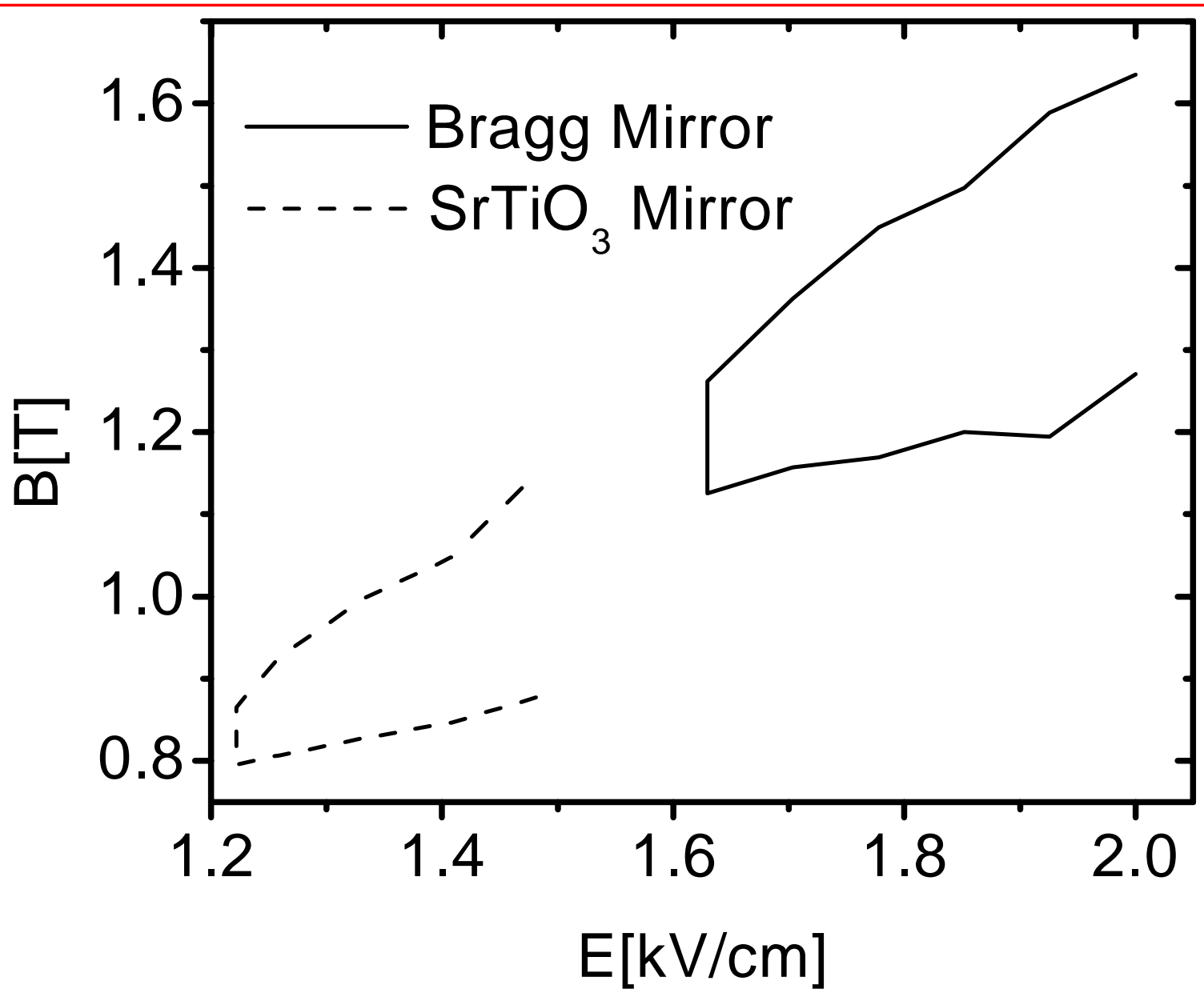

Fig. 4. Comparison of the generation zones using Bragg and $\mathrm{SrTiO}_{3}$ back mirrors.

\section{INTERPRETATION}

The far-infrared multi-layer Bragg mirror has sufficient reflection for use in the $\mathrm{p}$-Ge laser. For a laser, the mirror reflectivity $R$ satisfies the condition

$$
R \geq e^{-2 \alpha L_{G e}}
$$

where $\alpha$ is the gain and $L_{G e}$ is the length of the Ge laser cavity. Using $0.01 \mathrm{~cm}^{-1}$ as a characteristic value for the gain of a p-Ge laser and $3.52 \mathrm{~cm}$ for the length of the laser crystal, the minimum reflectivity for a laser mirror is $\mathrm{R}_{\min } \sim$ $93 \%$. Output coupling losses require the reflectivity to be better than $93 \%$. $\mathrm{SrTiO}_{3}$ has a reflectivity of $\sim 99 \%$ at $4 \mathrm{~K}$ in this wavelength region. ${ }^{10}$ The higher threshold for the Bragg mirror therefore suggests that its reflectivity is somewhat lower than 99\%. Hence, this simple experiment confirms that the reflectivity of the Bragg mirror is quite high, though it appears to fall short of the predicted $99.9 \%$ reflectivity.

We note that the Bragg mirror tested is a complicated and unstable structure. The silicon pieces are very thin and difficult to handle. Maintaining parallelism during mirror assembly and clamping to the Ge laser rod is probably not completely successful. From Figure 3, it is clear that the brass washer and Si stack supports cover portions of the active crystal which might support laser modes, though these regions of the mirror would not have the high design reflectivity. A more monolithic construction can be made by wet chemical etching to produce the gaps, ${ }^{11}$ eliminating the need for the small silicon spacers. 


\section{CONCLUSIONS}

A multi-layer Bragg mirror was demonstrated as a cavity mirror for the far-infrared $\mathrm{p}$-Ge laser. The initial test shows the reflectivity lower than the expected $99.9 \%$ but the reflectivity is nevertheless sufficiently high to be used as a cavity mirror for the low gain p-Ge laser. Improvements to the construction of the Bragg mirror may increase its reflectivity toward the predicted values. This might enable the Bragg mirrors to be used in a CRDS system.

\section{ACKNOWLEDGEMENTS}

This work was partially supported by NSF grant ECS-0070228 to UCF and AFOSR F49620-02-C-0027 STTR Phase II to Zaubertek.

\section{REFERENCES}

1) Cavity-Ringdown Spectroscopy - An Ultratrace-Absorption Measurement Technique K. W. Busch and M. A. Busch, eds., (American Chemical Society/Oxford University Press, 1999).

2) “Cavity Ring Down Spectroscopy: Experimental schemes and applications," G. Berden, R. Peeters, and G. Meijer, Intl. Rev. Phys. Chem. 19, 565 (2000).

3) Far Infrared Spectroscopy, K.D. Möller and W.G. Rothschild (Wiley, New York, 1971) p. 92.

4) "Etalon, lamellar, and Bragg intracavity wavelength selecting mirrors for the far-infrared p-Ge laser," T.W. Du Bosq, E.W. Nelson, A.V. Muravjov, D.A. Walters, G. Subramanian, K.B. Sundaram, R.E. Peale, N. Tache, D.B. Tanner, and C.J. Fredricksen, in Optics in the Southeast 2003 Program (Optical Society of America, Wash. D.C., Nov 2003), PSE 01-6.

5) "Far-infrared multilayer mirrors," R. Schiwon, G. Schwaab, E. Bruendermann, and M. Havenith, Appl. Phys. Lett. 83, 4119 (2003).

6) "Widely Tunable Far-Infrared Hot-Hole Semiconductor Lasers," Eric Brundermann in Long-Wavelength Infrared Semiconductor Lasers, edited by Hong K. Choi (Wiley, New Jersey, 2004) Chapter 6.

7) "Optical Properties of Films and Coatings," J.A. Dobrowolski in Handbook of Optics:fundamentals, techniques, $\&$ design Vol I, Michael Bass editor in chief (McGraw-Hill, New York) 42.10 (1995).

8) “Optical constants of far infrared materials 2: Crystalline solids," E. W. Loewenstein, D. R. Smith, and R. L. Morgan, Appl. Opt. 12, 398 (1973).

9) "High resolution study of composite cavity effects for p-Ge lasers," E. W Nelson, S. H. Withers, A. V. Muravjov, R. C. Strijbos, R. E. Peale, S. G. Pavlov, V. N. Shastin, and C. J. Fredricksen, IEEE J. of Quantum Electronics 37, 1525 (2001).

10) "Dielectric selective mirror for intracavity wavelength selection in far-infrared p-Ge lasers," T.W. Du Bosq, R.E. Peale, E.W. Nelson, A.V. Muravjov, C.J. Fredricksen, N. Tache, and D.B. Tanner, J. Appl. Phys. 94, 5474 (2003).

11) "Wavelength selection for the far-infrared p-Ge laser using etched silicon lamellar gratings," T. W. Du Bosq, R. E. Peale, E. W. Nelson, A. V. Muravjov, D. A. Walters, G. Subramanian, K. B. Sundaram, and C. J. Fredricksen, Optics \& Laser Technology, accepted (2004). 